\title{
Proto-Neutron Stars with Delta-Resonances Using the Zimanyi-Moszkowski Model
}

\section{V. S. Gomes*}

Secretaria de Educação, Cultura e Desportos do Estado de Roraima

Praça do Centro Cívico 471, 69301-380, Roraima, Brazil

E-mail: luzavilanova@yahoo.com.br

\section{J. C. T. Oliveira}

Departamento de Física, Universidade Federal de Roraima

Campus do Paricarana s/n, 69310-270, Roraima, Brazil

E-mail: jctoliveiraedfis.ufrr.br

\section{H. Rodrigues ${ }^{a b}$}

${ }^{a}$ Department of Physics, San Diego State University 5500 Campanile Drive, San Diego, CA 92182, USA

${ }^{b}$ Centro Federal de Educação Tecnológica do Rio de Janeiro Av Maracanã 249, 20271-110, Rio de Janeiro, RJ, Brazil

E-mail: hargesciences.sdsu.edu

\section{S. B. Duarte}

Centro Brasileiro de Pesquisas Físicas

Rua Dr. Xavier Sigaud 150, 22290-180, Rio de Janeiro, RJ, Brazil

E-mail: sbdecbpf.br

\begin{abstract}
In this work we have obtained the equation of state to be used in the study of the structure of protoneutron stars. To this end, we adopted the model of Zimanyi-Moszkowski (ZM) in the mean field approximation. We analyse the effects of the trapped neutrinos on the equation of state during the initial formation of a neutron star. We determine the Mass $\times$ Radius diagram of proto-neutron stars including the delta resonances in the baryonic sector and trapped neutrinos. The result is compared with that for the formed neutron star after the cooling phase, without trapped neutrino in the stelar medium.
\end{abstract}

XXXIV edition of the Brazilian Workshop on Nuclear Physics,

5-10 June 2011

Foz de Iguaçú, Paraná, Brazil

\footnotetext{
* Speaker.
} 


\section{Introduction}

The production of delta-resonances in the dense phase $\left(\rho \geq 3 \rho_{0}\right)$ of relativistic heavy ion collisions $[1,2,3]$, where $\rho_{0}$ is the normal nuclear matter density, leads to a great interest in the study of the delta matter formation in the interior of proto-neutron stars. In the present work the delta-rich hadronic condensate is studied in the context of a relativistic mean field calculation for hadrons in beta-equilibrium with the leptons present in the medium. The baryonic sector includes the complete $1 / 2$-spin octet and delta-resonances with 3/2-spin, which interact by exchange of scalar, vector and isovector mesons $(\sigma, \omega$ and $\rho)$. In the leptonic sector for the beta-equilibrium condition it was considered that the neutrinos were trapped in the stellar medium. The purpose here was to study the effects of neutrino trapping on the equation of state during the initial instants of neutron star formation.

\section{The Model}

We consider in the calculations the $1 / 2$-spin baryon octet $\left(n, p, \Lambda^{0}, \Sigma^{-}, \Sigma^{0}, \Sigma^{+}, \Xi^{-}, \Xi^{0}\right)$, the $3 / 2$-spin baryonic resonances represented by the delta-matter $\left(\Delta^{-}, \Delta^{0}, \Delta^{+}, \Delta^{++}\right)$and $\Omega^{-}$, in the baryonic sector [4, 5], and electrons, muons and trapped neutrinos in the leptonic sector. We have adopted the Zimanyi-Moszkowski (ZM) model [6] with the Lagrangian density given by $\mathscr{L}=$ $\mathscr{L}_{F}+\mathscr{L}_{I}$, where

$$
\begin{aligned}
\mathscr{L}_{F} & =\sum_{B} \bar{\Psi}_{B}\left(i \gamma_{\mu} \partial^{\mu}-M_{B}\right) \Psi_{B}+\sum_{\zeta} \bar{R}_{\zeta v}\left(i \gamma_{\mu} \partial^{\mu}-M_{\zeta}\right) R_{\zeta}^{v} \\
& +\frac{1}{2}\left(\partial_{\mu} \sigma \partial^{\mu} \sigma-m_{\sigma}^{2} \sigma^{2}\right)-\frac{1}{4} \omega_{\mu \nu} \omega^{\mu \nu} \\
& +\frac{1}{2} m_{\omega}^{2} \omega_{\mu} \omega^{\mu}-\frac{1}{4} \rho_{\mu \nu} \cdot \rho^{\mu v}+\frac{1}{2} m_{\rho}^{2} \rho_{\mu} \cdot \rho^{\mu} \\
& +\sum_{\lambda} \bar{\Psi}_{\lambda}\left(i \gamma_{\mu} \partial^{\mu}-m_{\lambda}\right) \Psi_{\lambda},
\end{aligned}
$$

is the Lagrangian density term for free baryons, electrons, muons and neutrinos $\left(\lambda=e^{-}, \mu^{-}, v_{e}\right.$, $\bar{v}_{\mu}$ ), and $\sigma, \omega$ and $\rho$ meson fields. In addition, the interaction Lagrangian has the form

$$
\begin{aligned}
\mathscr{L}_{I} & =\sum_{B} \bar{\Psi}_{B}\left(m_{B}^{*} g_{\sigma B} \sigma-g_{\omega B} \gamma_{\mu} \omega^{\mu}-\frac{1}{2} g_{\rho B} \gamma_{\mu} \tau \cdot \rho^{\mu}\right) \Psi_{B} \\
& +\sum_{\zeta} \bar{R}_{\zeta v}\left(m_{\zeta}^{*} g_{\sigma \zeta} \sigma-g_{\omega \zeta} \gamma_{\mu} \omega^{\mu}-\frac{1}{2} g_{\rho \zeta} \gamma_{\mu} \tau \cdot \rho^{\mu}\right) R_{\zeta}^{v} .
\end{aligned}
$$

In the equations above the operator $\Psi_{B}$ represents the Dirac spinor describing the baryon-octet, with $B=n, p, \Lambda^{0}, \Sigma^{-}, \Sigma^{0}, \Sigma^{+}, \Xi^{-}, \Xi^{0}$. The operator $R_{\zeta v}$ is the Rarita-Schwinger spinor [7], with $\zeta=\Delta^{-}, \Delta^{0}, \Delta^{+}, \Delta^{++}, \Omega^{-}$. The effective baryon mass $M_{i}^{*}$ for this model is defined by $M_{i}^{*}=M_{i}-$ $m_{i}^{*} g_{\sigma i} \sigma$, where $m_{i}^{*}=\left(1+\frac{g_{\sigma i} \sigma}{M_{i}}\right)^{-1}$.

In the present work we have varied the quantities $\alpha=g_{\omega \Delta} / g_{\omega N}, \quad \beta=g_{\sigma \Delta} / g_{\sigma N}$ and $\gamma=$ $g_{\rho \Delta} / g_{\rho N}$. For all the analysis we have used the set of coupling constants given in Ref. [6], namely $\left(g_{\sigma N} / m_{\sigma}\right)^{2}=7.487 \mathrm{fm}^{2},\left(g_{\omega N} / m_{\omega}\right)^{2}=2.615 \mathrm{fm}^{2},\left(g_{\rho N} / m_{\rho}\right)^{2}=4.774 \mathrm{fm}^{2}$. 


\section{Results and Conclusions}

Using the equation of state obtained by the model described in the last section, we have numerically solved the Tolmann-Oppenheimer-Volkoff (TOV) structure equations $[8,9]$ in order to obtain the mass-radius relationship.

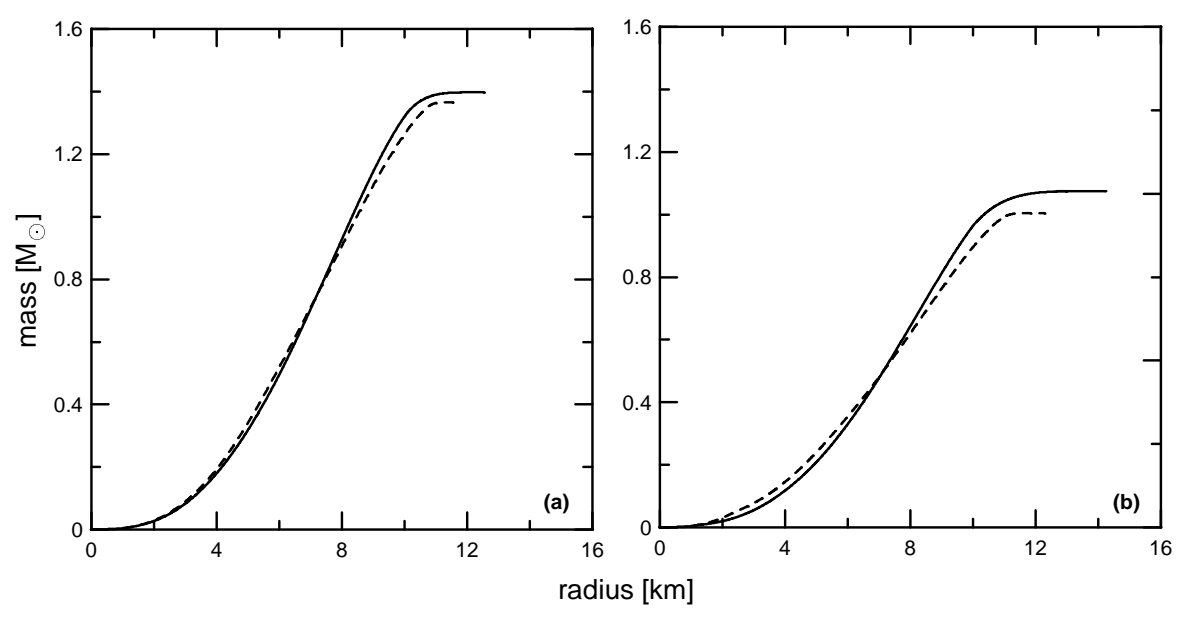

Figure 1: Mass-radius diagram obtained for the cases $\alpha=0.6, \beta=1.0, \gamma=1.0$ (part-a) and $\alpha=0.6, \beta=$ $1.4, \gamma=1.0$ (part-b) with (solid lines) and without (dashed lines) neutrinos. The figure shows the results for the central density $2.0 \times 10^{15}\left(\mathrm{~g} / \mathrm{cm}^{3}\right)$.

The Figure 1 shows the obtained mass-radius diagrams for the sets of parameters $\alpha=0.6, \beta=$ $1.0, \gamma=1.0$ and $\alpha=0.6, \beta=1.4, \gamma=1.0$ with and without neutrinos. In conclusion, we remark that the proto-neutron star has a maximum mass higher that the neutron star, since it has trapped neutrinos, as can be seen in the figure. Trapped neutrinos tend to stiffen the equation of state, and thus this explains why the newborn proto-neutron star can support more matter against the gracitational field, in comparison with a neutron star.

\section{References}

[1] E. L. Hjort et al, Phys. Rev. Lett. 79 (1997) 4345.

[2] M. Hofmann, R. Mattiello, H. Sorge, H. Stöcker and W. Greiner, Phys. Rev. C51 (1995) 2095.

[3] B. Hong et al., Phys. Lett. B407 (1997) 115. 
[4] J. C. T. de Oliveira, M. Kyotoku, M. Chiapparini, H. Rodrigues and S. B. Duarte, Mod. Phys. Lett. A15 (2000) 1529.

[5] J. C. T. de Oliveira, S. B. Duarte, H. Rodrigues, M. Chiapparini and M. Kyotoku, Int. J. Mod. Phys. D16 (2007) 175.

[6] J. Zimanyi and S. A. Moszkowski, Phys. Rev. C42 (4) (1990) 1416.

[7] W. Rarita and J. Schwinger, Phys. Rev. 60 (1941) 61.

[8] R. C. Tolman, Phys. Rev. 55 (1939) 364.

[9] J. R. Oppenheimer and G. M. Volkoff, Phys. Rev. 55 (1939) 374. 